\section{Dilated examination of patients referred with minor lid complaints - is it necessary?}

\begin{abstract}
Objective To evaluate whether dilated fundus examination is necessary on patients presenting to clinic with lid complaints and normal vision.

Methods Patients with lid complaints were recruited from general and emergency clinics. Patients with visual symptoms or previous ophthalmic history were excluded. Subjects were examined by a junior ophthalmologist with slit-lamp biomicroscopy and Goldmann tonometry. Dilated posterior segment examination was performed with a Volk lens and the peripheral retina was examined with a three-mirror contact lens.
\end{abstract}

Results A total of 100 patients (200 eyes) were recruited, 63 females and 37 males with an average age of 45 years (SD of 19 years). The majority of lid abnormalities were chalazia (66) and papilloma (21). Posterior segment findings were early cataracts in five cases (eight eyes), macular drusen in three cases (five eyes), peripheral retinal lattice degeneration in two cases (three eyes), retinal pigment epithelial changes in one case (two eyes), a choroidal naevus in one eye, choroidal atrophy in one eye, and one case with asymmetric disc cupping. Six patients were seen by senior ophthalmologists and all were discharged after the first visit.

Conclusions In our sample of $\mathbf{1 0 0}$ patients presenting with lid complaints and normal visual acuity, dilated examination revealed no sight-threatening conditions that required further treatment or regular follow-up. Therefore, a single episode of screening for nonspecified retinal disease in a group with no particular risk factors is an inefficient screening method.

Eye (2007) 21, 941-942; doi:10.1038/sj.eye.6702348; published online 28 April 2006
PJ Tomlins' ${ }^{1}$ S Benskin², M Tahhan² and AK Tyagi ${ }^{1,2}$

Keywords: screening; chalazion; fundoscopy; dilation

\section{Introduction}

Thorough examination is central to ophthalmology; however, when is a particular examination unnecessary, time consuming, and irksome for the patient? Is significant pathology uncovered during dilated fundus examination?

\section{Materials and methods}

We recruited patients with lid complaints and excluded those with visual symptoms or previous ophthalmic history.

\section{Results}

A total of 100 patients (200 eyes) were recruited, 63 females and 37 males with an average age of 45 years (SD 19 years). Potentially significant findings on dilated examination were a choroidal naevus and one patient with asymmetric disc cupping. No patient had raised intraocular pressures. Six patients were referred for consultant opinion and all were immediately discharged.

\section{Discussion}

Wilson's criteria ${ }^{1}$ are to assess screening programmes and state that a screening programme must be a continuous process within an at-risk population. Clearly, a single examination of a group of mixed patients looking for any condition is not a continuous process screening an 'at-risk' population.

Wilson's criteria also state, that the condition screened for must have an understood disease progress with a detectable premorbid stage.
${ }^{1}$ Birmingham and Midlands Eye Centre, City Hospital, Birmingham,

West Midlands, UK

${ }^{2}$ Ophthalmology Department, Sandwell General Hospital, Lyndon, West Bromwich, West Midlands, UK

Correspondence: PJ Tomlins, Department of Ophthalmology,

Birmingham and Midlands Eye Centre,

City Hospital, Dudley Road, Birmingham,

West Midlands

B18 7QH, UK

Tel: 0121554 3801;

Fax: 01215543801

E-mail: paultomlins@mac.com

Received: 20 August 2005 Accepted in revised form: 20 February 2006 Published online: 28 April 2006 
Patients with lid complaints represent a heterogeneous group and may potentially suffer from many diseases, which may not fulfill Wilson's criteria.

One may wish to screen those patients with identifiable risk factors, for example diabetics. However, such individuals may already be in a screening programme, and so may be screened unnecessarily. Furthermore, screening may be carried out more effectively with a formal programme with regular screening.

Clinicians worry about missing life-threatening conditions. However, a retrospective study by Bove and Char $^{2}$ found $37 \%$ of patients with known uveal tumours had been examined within the past year by a general ophthalmologist and no malignancy detected.

A retrospective study by Pollack and Brodie ${ }^{3}$ looked at dilated fundus findings of asymptomatic patients. They found similar lesions to those in our prospective sample of patients with lid complaints.

\section{Conclusions}

Both of the potentially significant findings could have conceivably been picked up on undilated examination. Our sample suggests that routine dilated examination of patients with lid complaints does not reveal significant pathology. In fact, by only examining the lids, these patients may be spared an unnecessary and inconvenient procedure.

\section{References}

1 Wilson JM, Jungner YG. Principles and Practices of Screening for Disease. World Health Organisation: Geneva, 1968.

2 Bove R, Char DH. Nondiagnosed uveal melanomas. Ophthalmology 2004; 111(3): 554-557.

3 Pollack AL, Brodie SE. Diagnostic yield of the routine dilated fundus examination. Ophthalmology 1998; 105(2): 382-386. 